Historic, Archive Document

Do not assume content reflects current scientific knowledge, policies, or practices. 



\section{HORNBERGER'S GLADIOLUS}

\section{SPECIAL FALL AND WINTER LIST \\ 1931-1932}

\section{F. C. HORNBERGER}

\section{CLARK STREET}

\section{HAMBURG, NEW YORK}

Prices quoted here are for spring delivery. These prices cancel all former quotations. We dig late and like our stock well cured before shipping to our customers. We will acept otders fur Fall delivery; shipments not to be made before November 15 th.

\section{OUR 1932 CATALOG}

Will be issued later, perhaps after January 1, 1932. This fall list is somewhat condensed and will not contain all the new varieties that will be listed in our large Annual Catalog. Early in the season we can only make our prices in line with what other leading growers are charging. So this season, in addition to our regular discounts and our well known liberal custom of overcounting on all orders, we will issue to each customer who sends us an order A SPECIAL ADVERTISING DISCOUNT COUPON. This will be as good as cash, to be used at any time within 18 months from date of issue, and will be accepted the same as cash by us, to its full face value, for HORNBERGER'S ORIGINATIONS ONLY, of which there are now over fourteen, representing some of the very finest Gladiolus grown.

\section{HOW OUR PLAN WORKS}

We intend to conduct an extensive advertising campaign this season, and instead of spending thousand of dollars with publishers, we intend to give the bulk of this value direct to our customers. We know that our customers have given us our very best advertising in the past and we wish to prove our appreciation. So this season for every penny or dollar you send us in cash, we are going to give back to you a very liberal part of what you send us in the form of "OUR SPECIAL ADVERTISING DIS. COUNT COUPON." Below is a sample coupon; do not cut this out. It is printed mainly to show you what the original looks like. given above, on any Hornberger Originations as listed below. Valid only when signed by

us. Good Until, Date.

Signed

This coupon is entirely distinct and has no connection with our regular cash discounts, and our generous over count on all orders. You will get all of these the same as in other years, but in addition we will give you a very liberal "COUPON VALUE" as a part of our advertising fund. The coupons for this season will be accepted (only) for varieties originated by F. C. Hornberger, of which there are now over fourteen very outstanding and distinct "prize winners." Some of the leaders in their type or colors. 
Please note that the value of the coupon will be largely influenced by the particular items you may chance to order. For example, assuming we have at hand two $\$ 10.00$ orders, one of them consisting mainly of items that are very scarce or of items where the price is already at bed rock. The other consists mainly of items that we have in ample quanity, the results would be that the latter order would likely be given double the coupon value of the first order.

\section{THIS COUPON WILL ALSO BE USED TO ADJUST PRICES}

We will also use this same coupon to make price adjustments all through the selling season. As we are compelled to issue this list, so early in the season, we do not know at this early date how much others are likely to cut prices and in particular of our own list of originations, but with this "coupon" we will adjust all such price changes as they take place. This coupon will keep our prices right. You cannot lose by our methods.

SETS AND SPECIAL OFFERS; This coupon will also take care of all of these. You can order the items wanted knowing that you will get full value and then some. We can sell you high class stock as low as any other reliable grower, and intend to do so. Some growers fill half their catologs with such offers. Our "Coupons" will take care of all of this and we guarantee we will give you as large value and as fine well grown stock as any other can furnish you at any price. Make up your own "Sets" and get free coupon.

\section{REGULAR DISCOUNTS FOR CASH IN FULL WITH ORDER}

On orders of $\$ 5.00$ to $15.00-10 \%$ in extra stock, your selection.

On orders of 15.00 to $40.00-15 \%$ in extra stock, your selection.

On orders of 40.00 to $100.00-20 \%$ in extra stock, your selection.

\section{OUR BULB QUALITY THE FINEST EVER}

We have had one of the best seasons in years. Bulbs all grown on new land and the quality and size are wonderful. Our cut flower production this season has surpassed all past records.

\section{OUR SPECIAL FALL PRICES}

This Fall and Winter List, while not our complete catalog, has been issued in answer to the great many requests that we receive for new 1932 prices. Our new Annual catalog will be issued early in January and will be a complete Guide with very much valuable information. The prices in this list cancel all other prices. We are sending out all the surplus of our old 1931 catalogs, as far as they go, to be used (only) for variety discription and other information. Disregard prices in old catalog if you do not receive one with this list. It will indicate that our supply is exhausted. We will quote bulblets per qt. or peck; ask for prices.

TERMS: Are cash or C. O. D. Or if you send us $20 \%$ of amount of order we will hold for spring delivery, cash before shipment. All orders from this list are prepaid, except orders for less then $\$ 1.00$ on which there is a postage and packing charge of 25c extra. No single item sold for less then 20c. Please order enough of each size so all your items will total $20 \mathrm{c}$ or more. 25 bulbs or bulblets sold at the 100 rate price; pints and half pints at the quart rate price; 3 bulbs at the 10 rate price. For less then 25 bulbs at 100 rate price add ten per cent to price. For 1000 rate multiply the 100 rate by $9 ; 250$ bulbs sold at 1000 rate prices. 3 or more bulbs at 100 rate price, plus $10 \%$.

ABBREVIATIONS USED: Are as follows; $(\mathrm{FCH})$ after a variety name indicates the variety was originated by F. C. Hornberger. Other abbraviations used are : (A) Austin) (B) Brown; (Bet) Betscher; (Brig) Briggs Floral Co; (Bill) Gladiolus Bill; (JC) Joe Coleman; (D) Diener; (Dec) Decorah Gardens; (Gel) Gelser Bros; (Hol) Holland variety; (Aust) Australian; (P) Palmer; (WP) Pfitzer; (Kin) Kingsley; (Sal) Salbach; (M) Miller; (S) Stevens; (V) Vaughan.

\begin{tabular}{|c|c|c|c|c|c|c|c|c|c|}
\hline & Quanity & 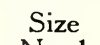 & I & 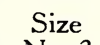 & NIEC & Size & Size & $\mathrm{Bul}$ & llets \\
\hline Variety & Per & No. 1 & No. 2 & No. 3 & No. 4 & No. 5 & No. 6 & Per & Price \\
\hline A. B. Kundred (K) & 100 & $\$ 8.00$ & $\$ 6.00$ & $\$ 4.00$ & $\$ 3.00$ & $\$ 2.00$ & $\$ 1.20$ & 1000 & $\$ 2.50$ \\
\hline Aflame $(\mathrm{FCH}) \ldots \ldots$ & 100 & 10.00 & 8.00 & 6.00 & 4.00 & 3.00 & 2.00 & 1000 & 2.00 \\
\hline Aida (WP) & 100 & 16.00 & 12.00 & 8.0 & 6.00 & 4.80 & 3.60 & 100 & 6 \\
\hline Albatross (WP) & 10 & 4.00 & 3.50 & 2.0 & 1.5 & & & & \\
\hline Anna Eberius (D) & 100 & 3.00 & 2.00 & 1.50 & 1.00 & .75 & .40 & 1 Qt. & 2.00 \\
\hline Laurie (B) & 100 & 4.00 & 3.20 & 2.50 & 1. & 1.00 & 65 & $1 \mathrm{Qt}$. & 2.5 \\
\hline t Glow $(P)$ & 100 & 4.5 & 3.5 & 2.5 & 2.00 & 1.5 & 1.00 & 1 & \\
\hline Aristocrat (Bill) & 100 & 6.00 & 5.00 & 4.00 & 3.00 & 2.0 & 1.00 & 1000 & 1.5 \\
\hline & 100 & 25.00 & 20.00 & 15.00 & 10. & 7. & 5.0 & 100 & 1.00 \\
\hline Snow (Mair) & 100 & 12.0 & 10.00 & 6. & 4. & 3. & 2.0 & 100 & .30 \\
\hline $\mathrm{Be}$ & 100 & 6.0 & 5.00 & 3. & 2. & & 1.2 & $1 \mathrm{Qt}$ & 3.0 \\
\hline thall ( & 100 & 15.0 & 12. & 8. & 6.00 & 5. & 3.00 & 1000 & 2.5 \\
\hline on & 1 & 2.50 & 2.00 & 1.5 & 1.00 & .75 & .50 & 10 & 2.5 \\
\hline & 1 & .3 & .4 & .3 & .25 & .20 & .15 & & \\
\hline Blue Torch $(\mathrm{FCH})$ & 100 & 5.00 & 4.00 & 3.00 & 2.00 & 1.50 & 1.00 & 1000 & 1.0 \\
\hline
\end{tabular}


Variety

Quanity Size

Blue Triumphator (WP).

Break O'Day (Bill)

Brown Wings $(\mathrm{FCH}) \ldots . . .$.

Buffalo (FCH)

Capt. Boynton (Rev. B) . ... 100

Cara Mia (M) .......... 10

Cardinal Prince $(\dot{K}) \ldots \ldots \ldots . . . .100$

Catherine Coleman (JC) . . . 100

Copper Bronze $(\mathrm{K}) \ldots \ldots \ldots . .100$

Coronado (Briggs) . . . . . . . 100

Coryphee (WP) ........... 100

Crimson Glow (B) . . . . ... 100

Crinkles (K)........... 100

Damascus (S)

100

Diener's Lilac (D)

Dr. F. E. Bennett (D) . . . . 100

Dr. Moody (Kinyon) ....... 100

Dr. Nelson Shook (K) . . . . . 100

Early Sunrise (Hol) ........ 100

Eliz. Swartley (SW) . . . . . . 100

Eliz. Tabor (Hinkle) . . . . . . . . 100

Ella Day (Car) ............ 10

Emile Auburn (L) .......... 100

Evelyn Kirtland (A) ........ 100

Florence (Auct)............ 100

Frank J. McCoy (Brig) ..... 10

Frederick Christ (D) . . . . . .

Geraldine Farrar (D) . . . . . 100

Giant Nymph (GC) . . . . . . 100

Gladdie Boy (Bill) . . . . . . . 10

Gloriana (Bet) ........... 100

Glory of U. S. A. (Cary) ....

Golden Dream (Groff) . . . . . 100

Golden Frills (K) . . . . . . . . 100

Golden Measure (Kel) . . . . . . 100

Grace Kimball (Dec)

H. Kanzleiter (Hol) . . . . . . , 100

Hamburg Pink (FCH) . . . . . 100

Heavenly Blue (WP) . . . . . . 100

Helen Phipps (Pety)....... 10

Iwa (B)

100

Ivanhoe $(\mathrm{FCH}) \ldots \ldots \ldots \ldots \ldots . . \ldots$

J. T. Pirie $(\mathrm{K}) \ldots \ldots \ldots \ldots \ldots$.

J. Van Beijeren (Hol) . . . . . . 100

Jane Adams (Dec) ......... 100

Jean du Tailles (L) . . . . . . . . 100

Jenny Lind (Dec) . . . . . . . . 100

Joerg's White $(\mathrm{J})$

Jubilee (Kemp)

Kirchoff's Violet. . . . . . . . 100

K's Yellow Wonder......... 100

La Paloma (Dusen) . . . . . . . . 10

Lavender Bride. . . . . . . . . . . 100

Loyalty $(\mathrm{A}) \ldots \ldots \ldots \ldots \ldots \ldots$

Lily of Gold . . . . . . . . . . .

Longfellow (Dec) . . . . . . . . 100

Mammoth White (Hol).....

Marmora (Erry) . . . . . . . . .

Mary Jane $(\mathrm{K})$

Matilda Ann (D) . . . . . .

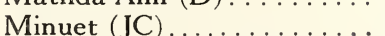

Miss Des Moines (Dec).....

Miss New York (FCH) . . . . .

Mother Machree (S) ........

Mr. W. H. Phipps (D) ......

No. 1

25.00

25.00

3.00

4.00

32.00

1.00

4.00

10.00

6.00

5.00

4.00

3.00

4.00

6.00

3.00

6.00

3.00

.75

7.00

3.00

1.00

4.00

2.50

5.00

5.00

4.00

4.00

.25

4.00

5.00

12.00

2.00

4.00

25.00

1.00

4.00

10.00

5.00

4.00

1.50

.75

6.00

5.00

20.00

10.00

32.00

1.00

4.00

1.00

Size

No. 2 Size

No. 3

Size

Size

Size

Bulblets

$\$ 8.00$

$\$ 6.00$

No. 4

3.00
1.00
5.00
3.50
2.00
4.00
6.50
4.00

2.00

.85

4.00

2.90

1.75

3.00

5.50

3.00

20.00

20.00

2.40

3.00

24.00

.75

3.00

8.00

5.00

4.00

3.00

2.00

3.00

5.00

2.00

5.00

2.50

.60

5.50

2.00

.80

3.00

2.00

4.00

4.00

3.00

3.00

.20

3.00

4.00

8.00

1.50

3.00

1.50

4.00

.70

3.00

2.40

1.50

2.50

4.50

2.00

15.00

15.00

2.00

2.40

20.00

.50

2.00

6.00

4.00

3.00

2.00

1.00

2.50

4.00

1.00

3.00

2.00

.50

4.00

1.00

.70

2.00

1.50

1.00

Sold

Sol

$50 \$$

Sold

1.20
.75

1.20

1.50

1.00

10.00

10.00

7.00

7.00

1.80

16.00

1.00

1.25

12.00

1.60

4.00

1.20

3.00

2.00

3.00

2.00

.85

2.00

1.50

2.50
75

1.50

2.00

1.00

1. 25

.80

2.00

.75

.60

1.00

2.40

3.00

2.00

2.40

3.00

5.00

1.00

.75

3.00

12.00

.50

8.00

2.00

4.00

6.00

3.00

2.50

1.25

2.00

.60

1.00
50

5.00

4.00

$4.00 \quad 3.00$

$16.00 \quad 12.00$

$8.00 \quad 6.00$

24.00

6.00
18.00

1.00

1.60

2.00

1.20

1.80

1.00

2.00

3.00

1.50

.50

.40

.80

1.20

1.00

1.00

.7

.75

1.50

2.00

.50

1.00 


\begin{tabular}{|c|c|c|c|c|c|c|c|c|c|}
\hline & Quanity & Size & Size & Size & Size & & Size & \multicolumn{2}{|c|}{ Bulblets } \\
\hline Variety & & & & No. 3 & & No. 5 & No. 6 & & Pric \\
\hline Mrs. Calvin Coolidge (K) & 100 & $\$ 10.00$ & $\$ 8.00$ & $\$ 5.00$ & $\$ 4.00$ & $\$ 3.00$ & $\$ 2.00$ & 1000 & $\$ 6.00$ \\
\hline Mrs. A. B. C. Dohrmann (D) & 100 & 6.00 & 5.00 & 4.00 & 3.00 & 2.00 & 1.00 & 1000 & 2.00 \\
\hline Leon Dougla & 100 & 4.00 & 3.00 & 2.00 & 1.60 & 1.00 & .80 & Qt. & 2.00 \\
\hline Mrs. Cath Edward & 100 & 40.00 & 32.00 & 24.00 & 16.00 & 12.00 & 8.00 & 1000 & 10.00 \\
\hline Irs. F. C. Hornberger (FCH) & ) 100 & 5.00 & 4.0 & 3.00 & 2. & & 1.00 & 1 Qt. & 2.00 \\
\hline Mrs. Dr. Norton $(\bar{K})$. & . 100 & 3.50 & 2.50 & 1.75 & 1.25 & 1.00 & .70 & $1 \mathrm{Qt}$. & 2.00 \\
\hline Irs. F. C. Peters (F) & . 100 & 4.00 & 3.00 & 2.00 & 1.60 & 1. & .80 & $1 \mathrm{Qt}$ & 1.50 \\
\hline P. W. Sisson (JC). & . 100 & 4.00 & 3.00 & 2.00 & 1.60 & 1.00 & .80 & $1 \mathrm{Qt}$. & 2.00 \\
\hline Van Konynenberg..... & . 100 & 7.00 & 5.6 & 3.60 & & & 1.00 & 1000 & 1.80 \\
\hline John Walsh (D) & 100 & 4.50 & 3.55 & 2.00 & 1. & 1. & .75 & 1000 & \\
\hline y $\operatorname{Han}$ & 100 & 4.00 & 3.00 & 2.40 & & & .80 & $1 \mathrm{Qt}$. & \\
\hline & 10 & 8.0 & 7.00 & 6.00 & 5. & 4.00 & 3.00 & 100 & \\
\hline . & 100 & & 2.7 & 2.00 & 1.5 & & & 1000 & \\
\hline & 100 & 3. & 2.5 & 1.00 & & & & 1 Qt. & \\
\hline . & . 100 & 20.00 & 16.00 & 10.00 & 8.00 & 6.00 & 4.00 & 100 & 1. \\
\hline & . 100 & 10. & 8.00 & 5.00 & 4.0 & 3.0 & 2.00 & 1000 & \\
\hline & 100 & 10 & 8. & 6.40 & 5.8 & 4.0 & 3.00 & 1000 & 7.00 \\
\hline K & 10 & & & & 2.0 & 1.5 & & 100 & \\
\hline n) $\ldots$. & 10 & 10.00 & $8 .($ & 6.00 & 4.00 & 3.0 & 2.00 & 1000 & \\
\hline & 100 & & 3.0 & 2.0 & & & & 1 Qt. & \\
\hline $\mathrm{Pf}$ & 100 & 10.00 & 8.00 & 5.00 & 4.00 & 3.00 & 2.40 & 1000 & \\
\hline$D$ & 100 & 5. & 4. & 3.0 & 2.25 & & & 1000 & \\
\hline$P$ & & 2. & 1. & 1.0 & .75 & .50 & .30 & 10 & \\
\hline & 100 & 4. & 3. & 2. & 2.00 & & & 1000 & \\
\hline & 100 & & & & .75 & .50 & 25 & 1Qt. & \\
\hline$(\mathrm{FCH})$ & 100 & $6 .($ & & 4. & 3.00 & 2.00 & 1.00 & 1000 & \\
\hline & 10 & 5. & & & 1.00 & .75 & .50 & 1000 & \\
\hline (D) & 100 & & & & 1.00 & .75 & .50 & 1000 & \\
\hline & 100 & 10. & & 5. & 4.0 & & & 1000 & \\
\hline( & 10 & & 2. & 1. & 1.8 & .75 & 1.00 & 100 & \\
\hline & 100 & 3. & 2. & 2. & 1.5 & 1.00 & . & $1 \mathrm{Qt}$. & \\
\hline & 10 & 4. & & & & & & 1000 & 1.0 \\
\hline & 10 & & & & 2. & & & 1000 & \\
\hline & 100 & 16 & 12 & 10. & 8. & 5.00 & 4.00 & 1000 & 8.0 \\
\hline & 10 & & & & . & .5 & .35 & 100 & \\
\hline & 10 & & & & & .90 & .75 & & 1. \\
\hline & 10 & 10.00 & 8. & 5. & 3.0 & 2.00 & 1.00 & 10 & \\
\hline & 10 & & & & & .2 & & 1000 & \\
\hline off). & 100 & & & 1. & 1. & .90 & & $1 \mathrm{Qt}$ & \\
\hline & 10 & & & 2. & & 1.00 & & 100 & \\
\hline & 10 & & & 2. & & & & 1Qt. & \\
\hline-1 & & & & & 1.4 & .9( & & 1000 & \\
\hline & 10 & & 2. & 2. & 1. & 90 & & i Qt. & 3. \\
\hline & & & & & .4 & 2 & .20 & & \\
\hline & 100 & & & & 1.6 & 1.2 & .80 & 1000 & \\
\hline & 100 & & & 3. & 2.4 & 1.60 & 1.00 & $1 \mathrm{Qt}$. & \\
\hline & & & & & & & & & \\
\hline $\mathrm{T}_{1}$ & 100 & 6. & 5. & $4 .($ & 3.0 & 2.00 & 1.00 & 1000 & \\
\hline & 10 & & & 2. & 1.5 & & & $1 \mathrm{Q}$ & 1. \\
\hline & 10 & 10. & & & & & & 1000 & \\
\hline & 100 & & & 12. & 9.00 & 7.00 & & 100 & 1.5 \\
\hline $\mathrm{CH}$ ) & 1 & & & & .75 & & & & \\
\hline & 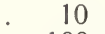 & & & & & & & & \\
\hline & 100 & 3. & & $3 .($ & 2.0 & 1.5 & 1.00 & $1 \mathrm{Qt}$ & \\
\hline Will & 100 & 5.0 & 4 & 3.0 & 2.00 & 1.50 & 1.00 & 1000 & \\
\hline
\end{tabular}

SPECIAL NOTICE: The 1000 rate will be from 7 to 9 times the 100 rate price. On itmes where stock is limited it will be nine times the hundred rate and on larger surplus lots it will run 7 to 8 times the 100 rate. But for the purpose of this prepaid price list figure all items at 9 times the 100 rate for the 1000 price and 250 at 1000 rate, and we will adjust all differences with our "Special Coupons" or if ycu prefer, we will quote you a special price on your larzer quantity orders.

\section{OUR TULIP LIST AND FALL PLANTING GUIDE}

Was mailed to you a few weeks ago. If you have mislaid your copy let us send you another. We have decided to apply our SPECIAL ADVERTISING COUPONS to all orders you send us, taken from this FALL PLANTING GUIDE. Take advantage of this "Special Discount on your Tulip and other Holland bulbs. 